SUPPORTING INFORMATION (Ms. ID: om-2016-00426q)

\title{
Amidinatogermylene Metal Complexes as Homogeneous Catalysts in Alcoholic Media
}

Lucía Álvarez-Rodríguez, Javier A. Cabeza,* José M. Fernández-Colinas,

Pablo García-Álvarez,* and Diego Polo

Departamento de Química Orgánica e Inorgánica-IUQOEM, Centro de Innovación en Química Avanzada (ORFEO-CINQA), Universidad de Oviedo-CSIC, E-33071 Oviedo, Spain

*E-mail: jac@uniovi.es (J.A.C.) and pga@uniovi.es (P.G.-A.) 
Table S1. Crystal, measurement and refinement data for the compounds studied by XRD

\begin{tabular}{|c|c|c|c|}
\hline & 2 & 5 & 6 \\
\hline formula & $\mathrm{C}_{27} \mathrm{H}_{44} \mathrm{ClGeN}_{2} \mathrm{Rh}$ & $\mathrm{C}_{29} \mathrm{H}_{46} \mathrm{Cl}_{2} \mathrm{GeN}_{2} \mathrm{Ru}$ & $2\left(\mathrm{C}_{29} \mathrm{H}_{47} \mathrm{Cl}_{2} \mathrm{GeIrN}_{2}\right)$ \\
\hline fw & 607.59 & 667.3 & 1518.75 \\
\hline cryst syst & Monoclinic & monoclinic & Monoclinic \\
\hline space group & $P 21 / \mathrm{c}$ & $\mathrm{P} 21 / \mathrm{n}$ & $P 21 / \mathrm{c}$ \\
\hline$a, \AA$ & $16.7872(2)$ & $9.2077(1)$ & $24.1428(2)$ \\
\hline$b, \AA$ & $12.2820(2)$ & $20.0025(4)$ & $9.3753(1)$ \\
\hline$c, \AA$ & $13.9392(2)$ & $16.7333(3)$ & $27.3016(2)$ \\
\hline$\alpha, \operatorname{deg}$ & 90 & 90 & 90 \\
\hline$\beta, \operatorname{deg}$ & $106.677(2)$ & $90.978(1)$ & $95.134(1)$ \\
\hline$\gamma, \operatorname{deg}$ & 90 & 90 & 90 \\
\hline$V, \AA^{3}$ & $2753.10(7)$ & $3081.44(9)$ & $6154.8(1)$ \\
\hline $\mathrm{Z}$ & 4 & 4 & 4 \\
\hline$F(000)$ & 1256 & 1376 & 3024 \\
\hline$D_{\text {calcd }}, \mathrm{g} \mathrm{cm}^{-3}$ & 1.466 & 1.438 & 1.639 \\
\hline$\mu, \mathrm{mm}^{-1}$ & $1.805(\mathrm{Mo} \mathrm{K} \alpha)$ & $6.900(\mathrm{Cu} \mathrm{K \alpha})$ & $11.185(\mathrm{Cu} \mathrm{K} \alpha)$ \\
\hline cryst size, $\mathrm{mm}$ & $0.19 \times 0.13 \times 0.10$ & $0.12 \times 0.10 \times 0.08$ & $0.26 \times 0.16 \times 0.09$ \\
\hline$T, \mathrm{~K}$ & $120.0(1)$ & $150(2)$ & $155(2)$ \\
\hline$\theta$ range, deg & 3.32 to 31.50 & 3.44 to 69.00 & 3.25 to 68.99 \\
\hline $\min . / \max . h, k, l$ & $-23 / 24,-17 / 18,-20 / 19$ & $-10 / 11,-23 / 23,-14 / 19$ & $-29 / 27,-8 / 11,-32 / 32$ \\
\hline no. collected reflns & 40344 & 14539 & 29901 \\
\hline no. unique reflns & 8550 & 5640 & 11357 \\
\hline no. reflns with $I>2 \sigma(I)$ & 7168 & 5094 & 10591 \\
\hline no. params/restraints & $298 / 0$ & $328 / 0$ & $659 / 0$ \\
\hline $\mathrm{GOF}\left(\right.$ on $F^{2}$ ) & 1.040 & 1.036 & 1.042 \\
\hline$R_{1}($ on $F, I>2 \sigma(I))$ & 0.031 & 0.043 & 0.024 \\
\hline$w R_{2}\left(\right.$ on $F^{2}$, all data $)$ & 0.063 & 0.108 & 0.059 \\
\hline $\min . / \max . \Delta \rho, \mathrm{e} \AA^{-3}$ & $-0.489 / 0.597$ & $-1.476 / 2.145$ & $-1.411 / 0.948$ \\
\hline CCDC dep. no. & 1480090 & 1480091 & 1480092 \\
\hline
\end{tabular}




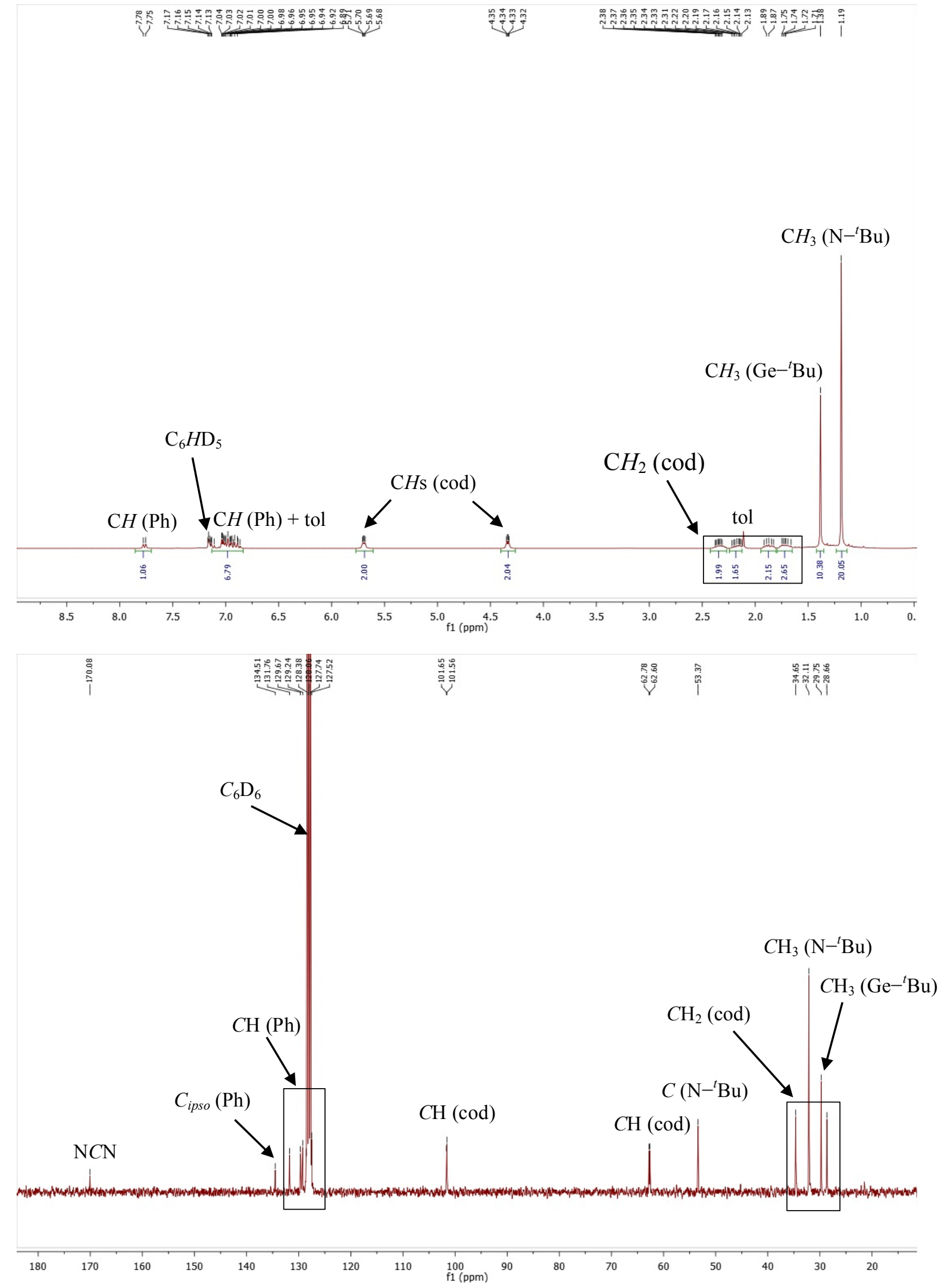

Figure S1. ${ }^{1} \mathrm{H}$ (top; $300.1 \mathrm{MHz}$ ) and ${ }^{13} \mathrm{C}\left\{{ }^{1} \mathrm{H}\right\}$ (bottom; 75.5 MHz) NMR spectra of $2\left(\mathrm{C}_{6} \mathrm{D}_{6}, 25{ }^{\circ} \mathrm{C}\right.$ ). 


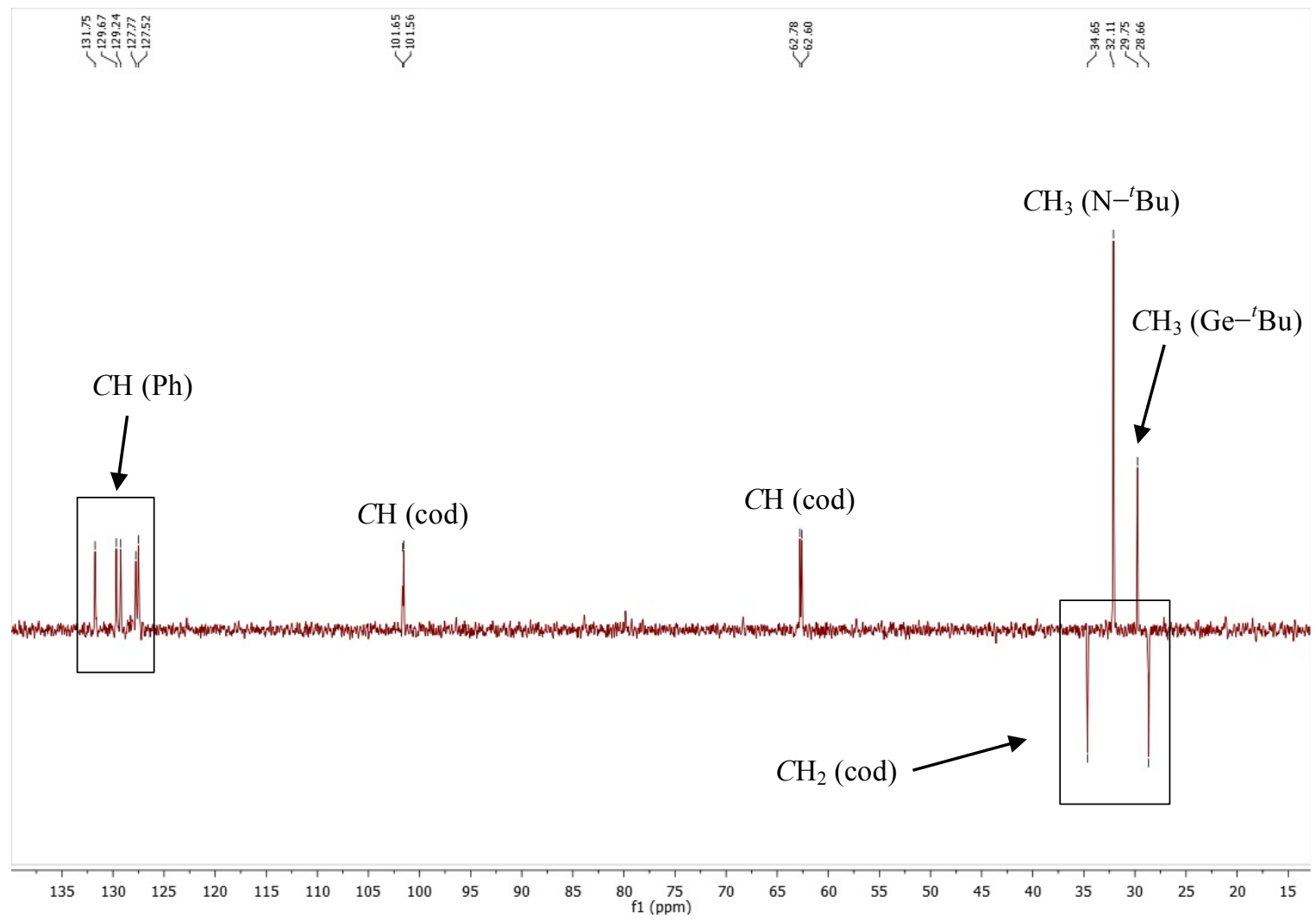

Figure S2. ${ }^{13} \mathrm{C}\left\{{ }^{1} \mathrm{H}\right\}$-DEPT $135(75.5 \mathrm{MHz})$ NMR spectrum of $2\left(\mathrm{C}_{6} \mathrm{D}_{6}, 25^{\circ} \mathrm{C}\right)$. 


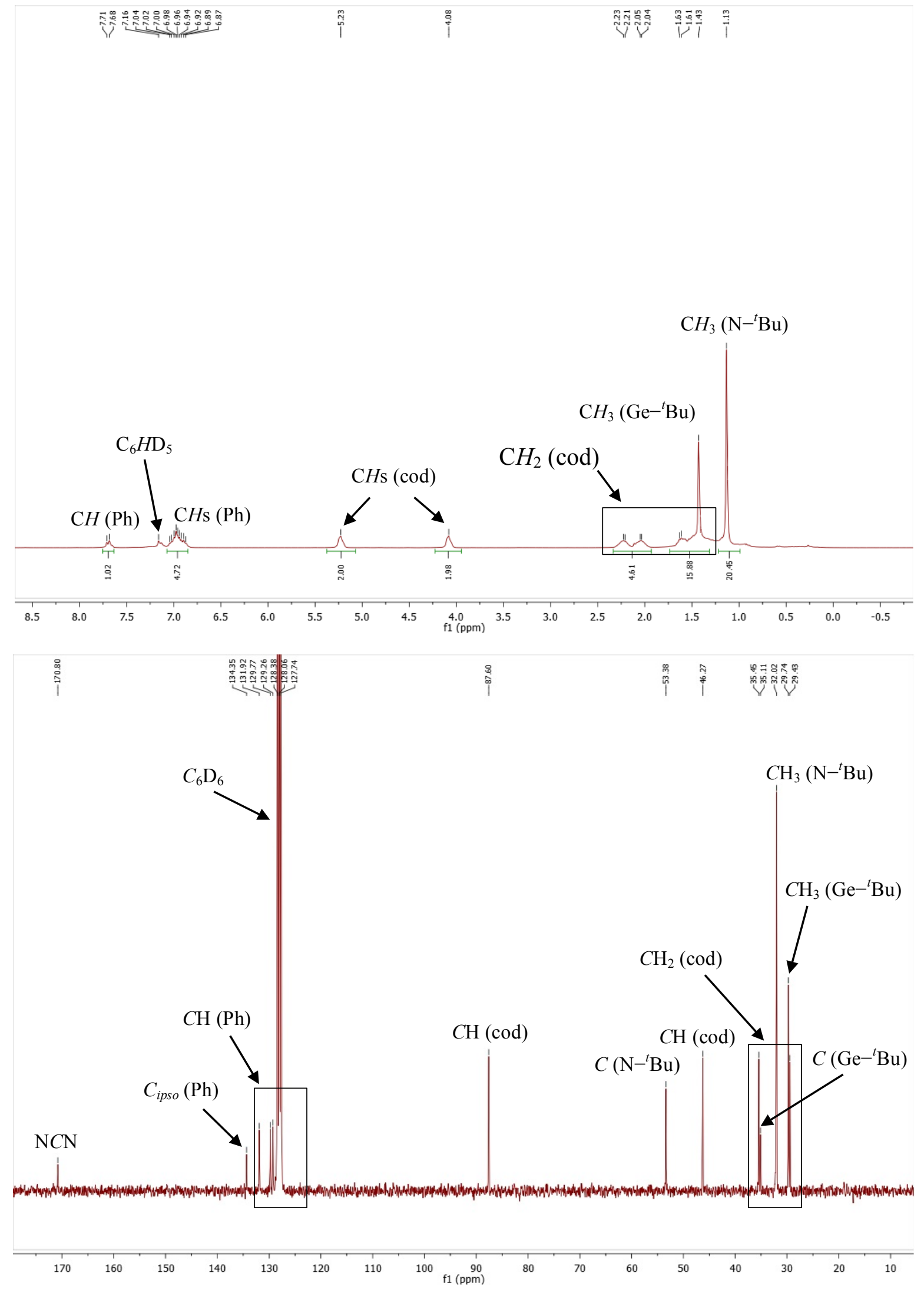

Figure S3. ${ }^{1} \mathrm{H}$ (top; $300.1 \mathrm{MHz}$ ) and ${ }^{13} \mathrm{C}\left\{{ }^{1} \mathrm{H}\right\}$ (bottom; $75.5 \mathrm{MHz}$ ) NMR spectra of $\mathbf{3}\left(\mathrm{C}_{6} \mathrm{D}_{6}, 25{ }^{\circ} \mathrm{C}\right.$ ). 


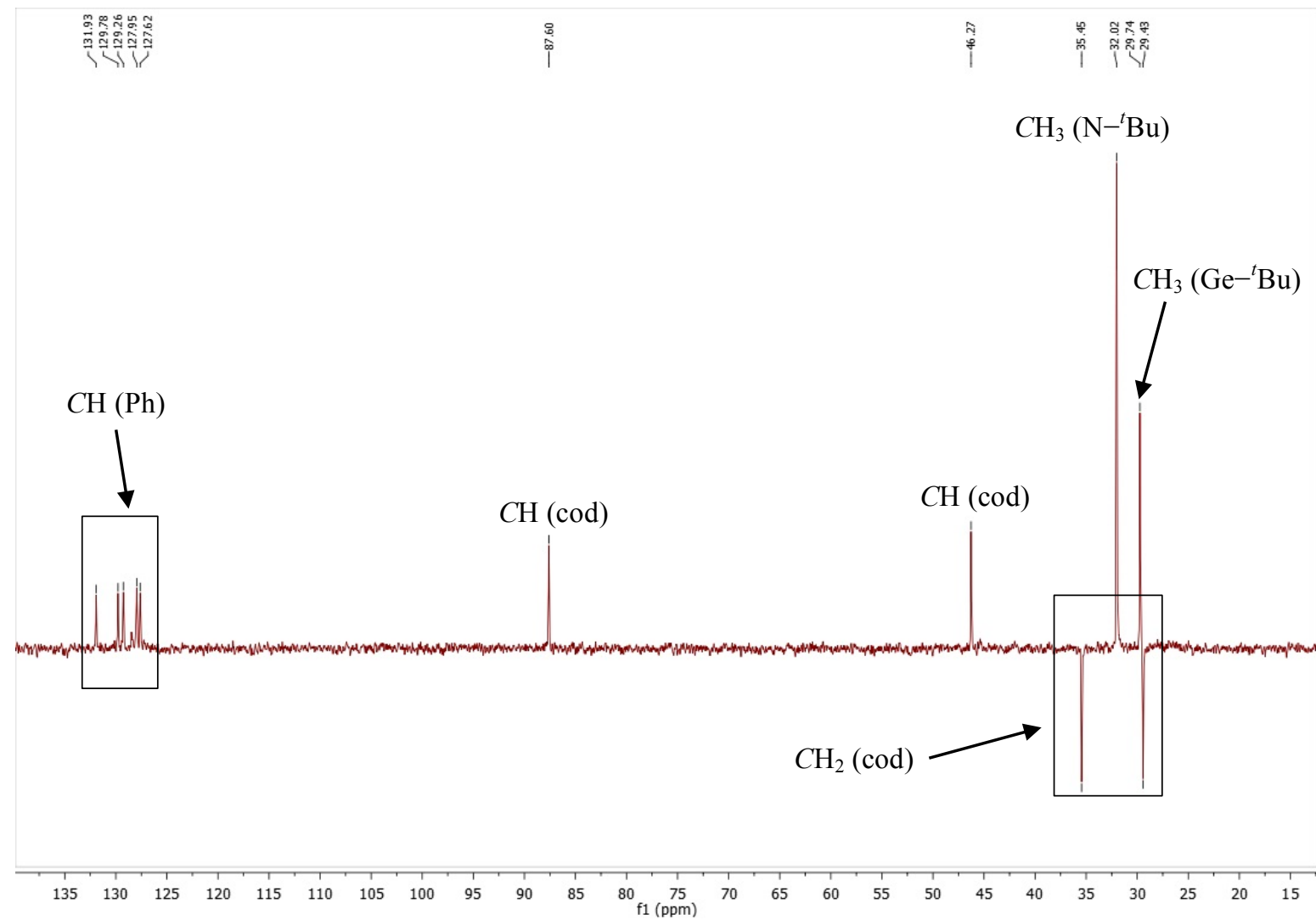

Figure S4. ${ }^{13} \mathrm{C}\left\{{ }^{1} \mathrm{H}\right\}$-DEPT $135(75.5 \mathrm{MHz}) \mathrm{NMR}$ spectrum of $\mathbf{3}\left(\mathrm{C}_{6} \mathrm{D}_{6}, 25{ }^{\circ} \mathrm{C}\right)$. 

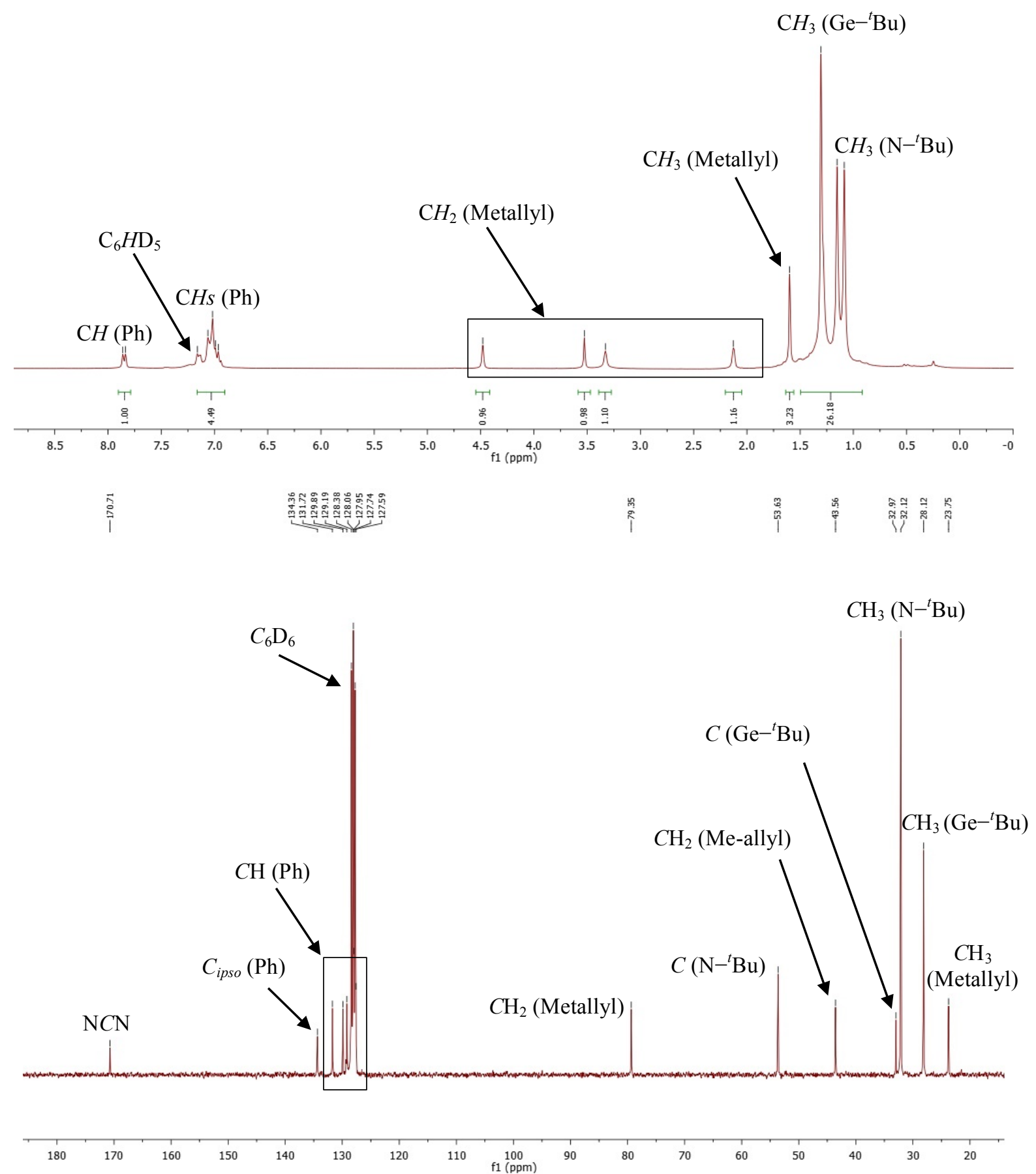

Figure S5. ${ }^{1} \mathrm{H}$ (top; $300.1 \mathrm{MHz}$ ) and ${ }^{13} \mathrm{C}\left\{{ }^{1} \mathrm{H}\right\}$ (bottom; $75.5 \mathrm{MHz}$ ) NMR spectra of $4\left(\mathrm{C}_{6} \mathrm{D}_{6}, 25{ }^{\circ} \mathrm{C}\right.$ ). 


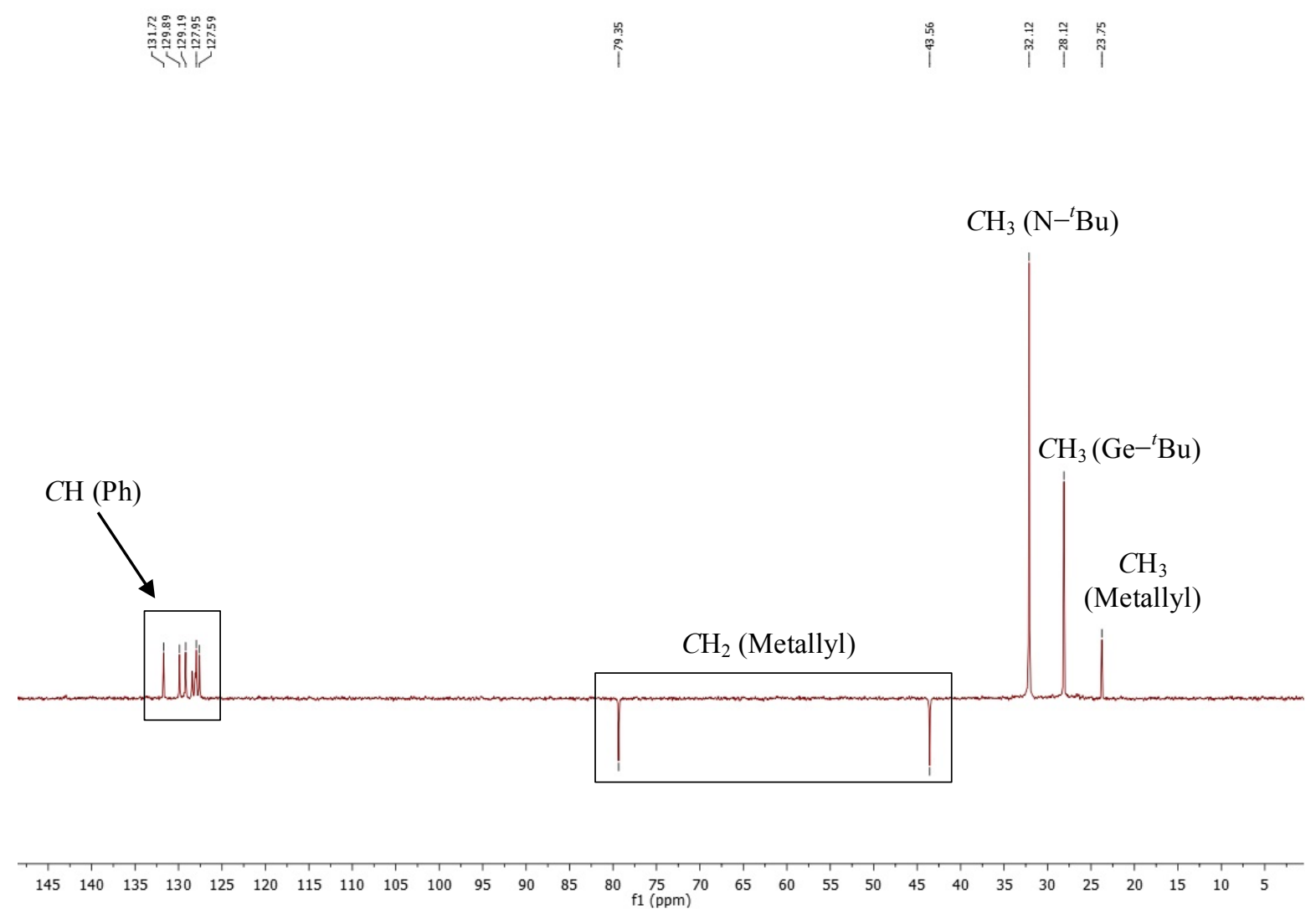

Figure S6. ${ }^{13} \mathrm{C}\left\{{ }^{1} \mathrm{H}\right\}$-DEPT $135(75.5 \mathrm{MHz})$ NMR spectrum of $4\left(\mathrm{C}_{6} \mathrm{D}_{6}, 25^{\circ} \mathrm{C}\right)$. 

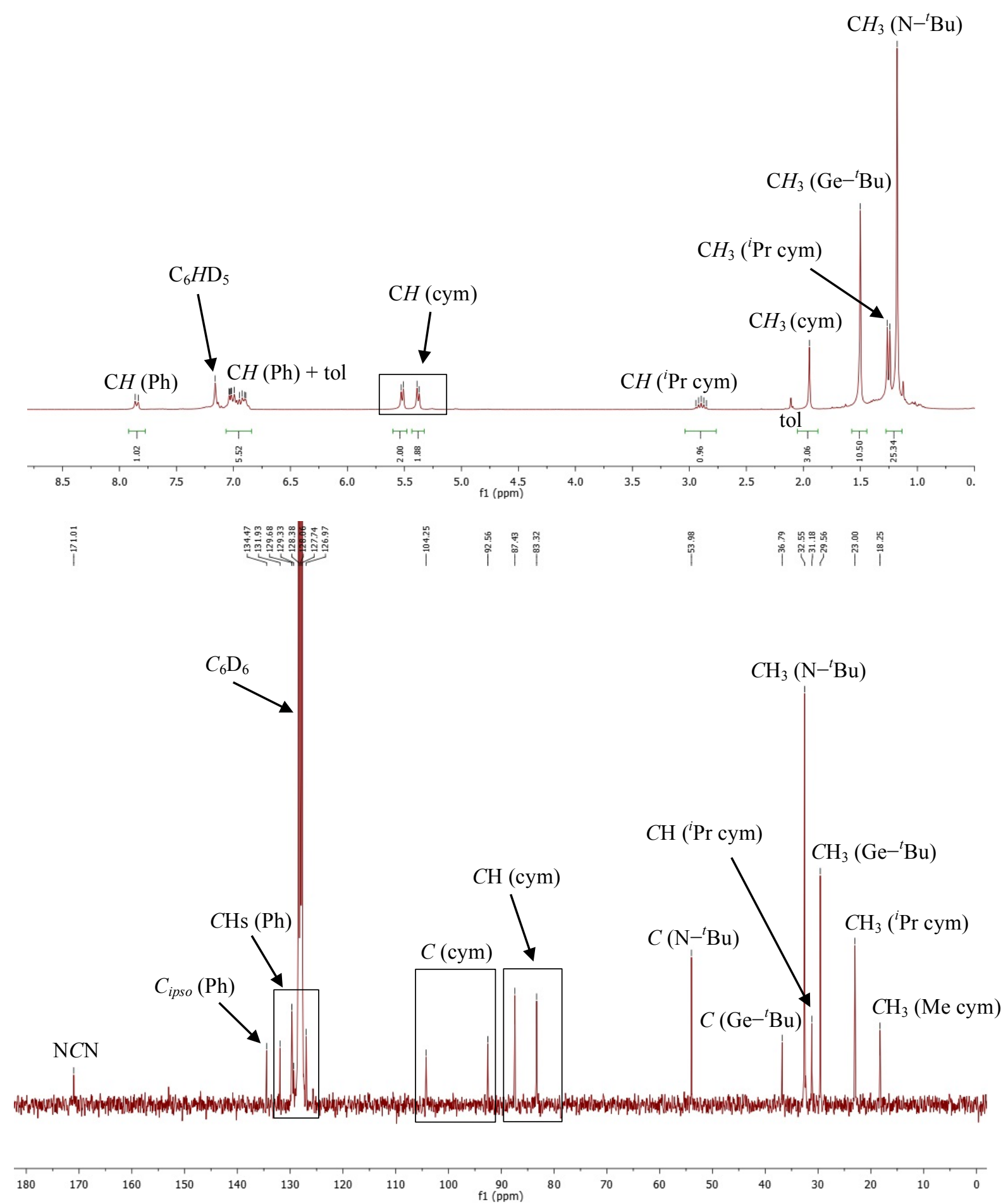

Figure S7. ${ }^{1} \mathrm{H}$ (top; $300.1 \mathrm{MHz}$ ) and ${ }^{13} \mathrm{C}\left\{{ }^{1} \mathrm{H}\right\}$ (bottom; $75.5 \mathrm{MHz}$ ) NMR spectra of $5\left(\mathrm{C}_{6} \mathrm{D}_{6}, 25{ }^{\circ} \mathrm{C}\right.$ ). 


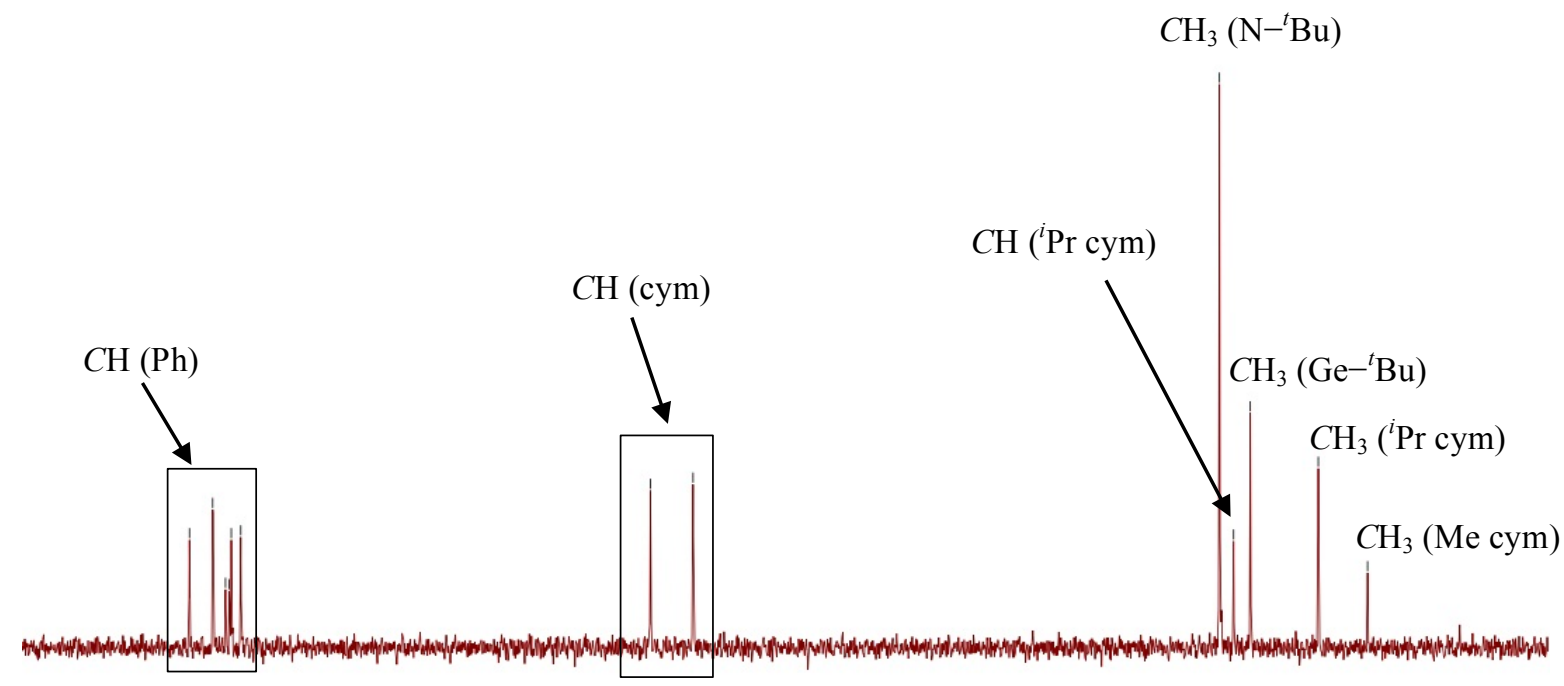

$\begin{array}{llllllllllllllllllllllllllllll}145 & 140 & 135 & 130 & 125 & 120 & 115 & 110 & 105 & 100 & 95 & 90 & 85 & 80 & 75 & 70 & 65 & 60 & 55 & 50 & 45 & 40 & 35 & 30 & 25 & 20 & 15 & 10 & 5\end{array}$

Figure S8. ${ }^{13} \mathrm{C}\left\{{ }^{1} \mathrm{H}\right\}$-DEPT $135(75.5 \mathrm{MHz}) \mathrm{NMR}$ spectrum of $\mathbf{5}\left(\mathrm{C}_{6} \mathrm{D}_{6}, 25^{\circ} \mathrm{C}\right)$. 

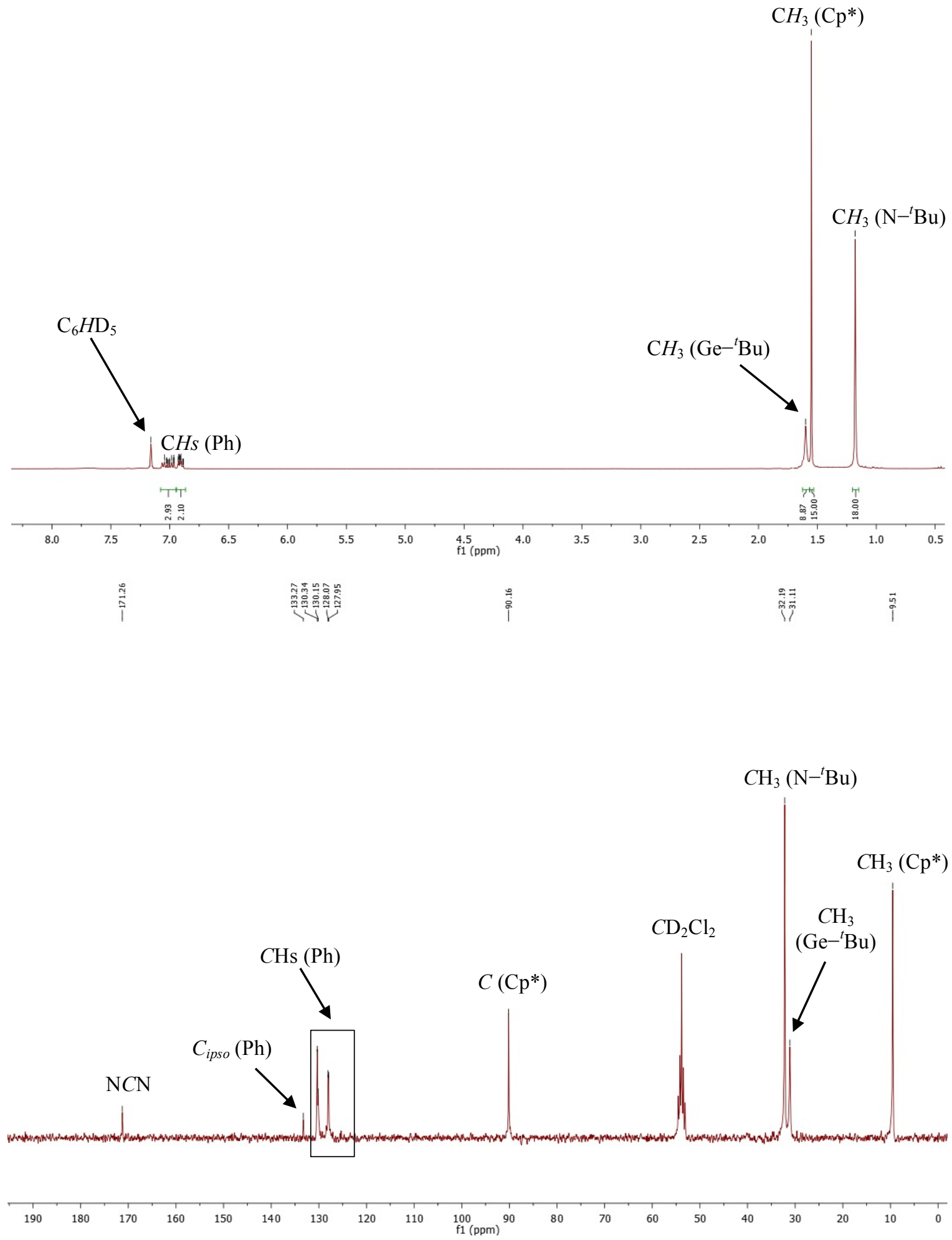

Figure S9. ${ }^{1} \mathrm{H}$ (top; $300.1 \mathrm{MHz}, \mathrm{C}_{6} \mathrm{D}_{6}$ ) and ${ }^{13} \mathrm{C}\left\{{ }^{1} \mathrm{H}\right\}$ (bottom; $\left.75.5 \mathrm{MHz}, \mathrm{CD}_{2} \mathrm{Cl}_{2}\right) \mathrm{NMR}$ spectra of $6\left(25{ }^{\circ} \mathrm{C}\right.$ ). 


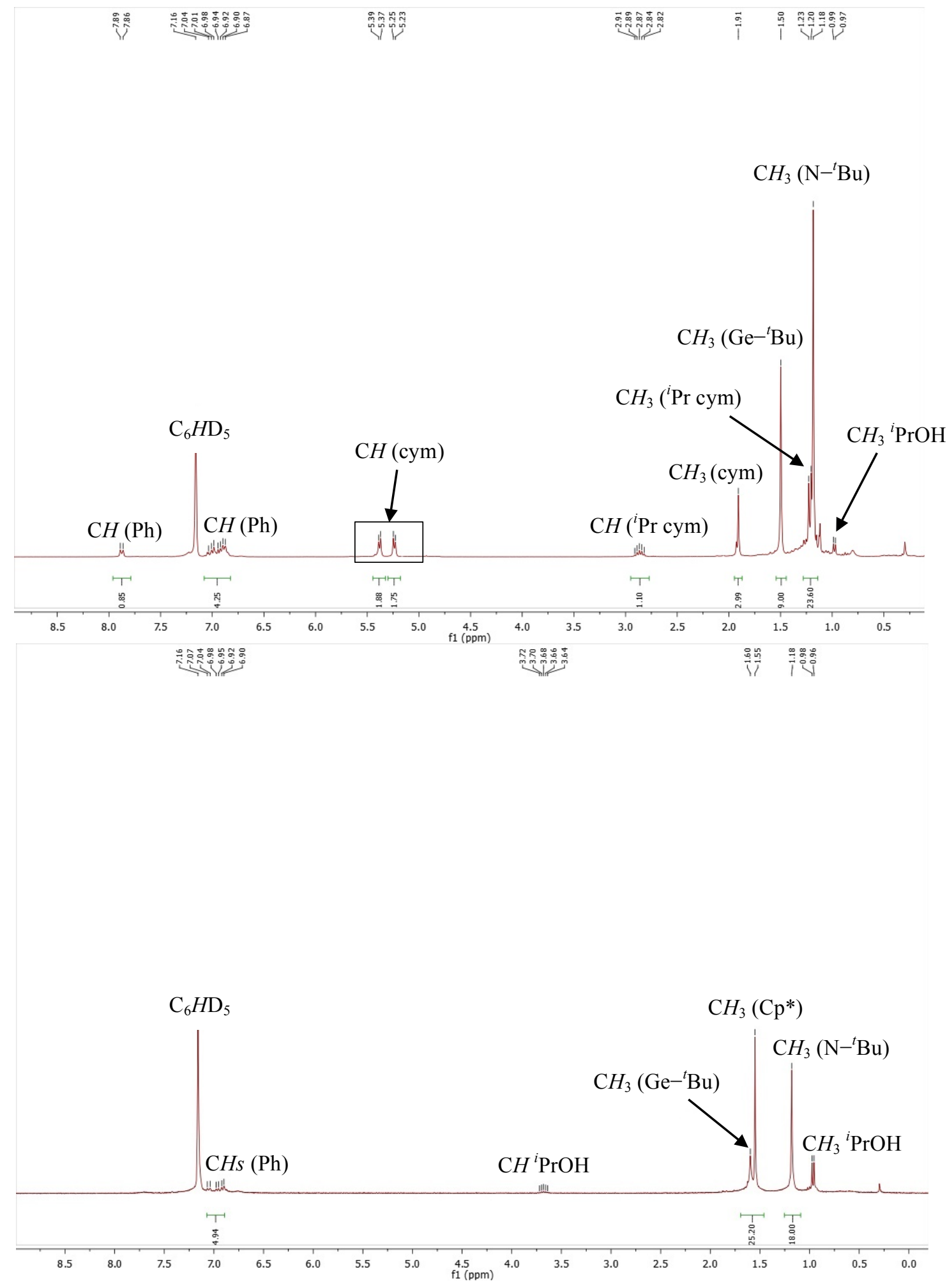

Figure S10. ${ }^{1} \mathrm{H}$ NMR spectra $\left(300.1 \mathrm{MHz}, \mathrm{C}_{6} \mathrm{D}_{6}, 25{ }^{\circ} \mathrm{C}\right)$ of $\mathbf{5}$ (top) and $\mathbf{6}$ (bottom) after one day in isopropanol under air. 

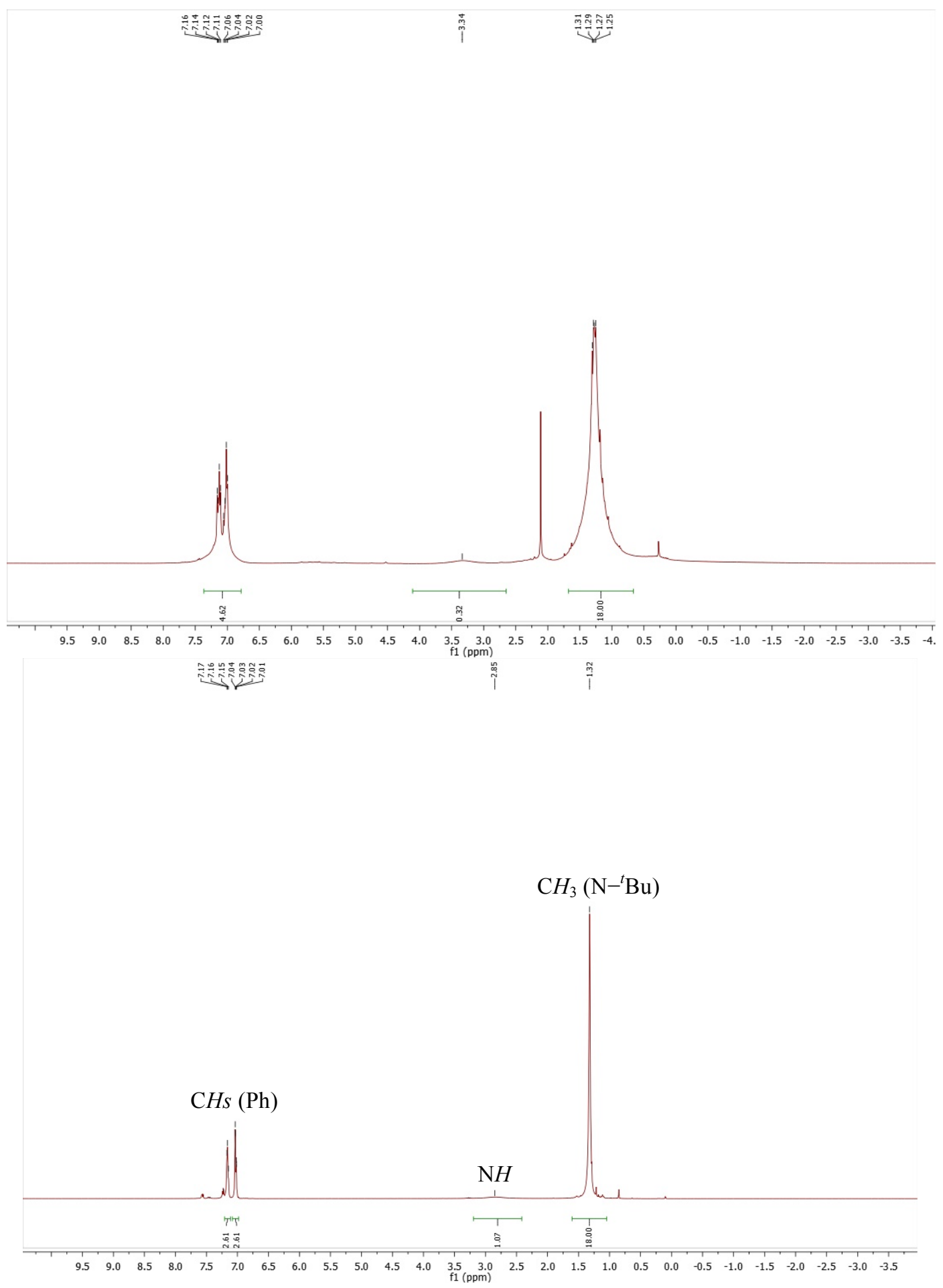

Figure S11. ${ }^{1} \mathrm{H}$ NMR spectra $\left(300.1 \mathrm{MHz}, \mathrm{C}_{6} \mathrm{D}_{6}, 25{ }^{\circ} \mathrm{C}\right)$ of 3 after one day in toluene under air (top) and of pure ${ }^{\mathrm{t}} \mathrm{Bu}_{2}$ bzamH (bottom). 


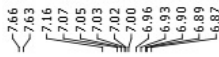

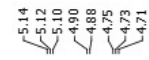

tol $^{*}=$ reaction solvent

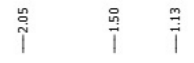

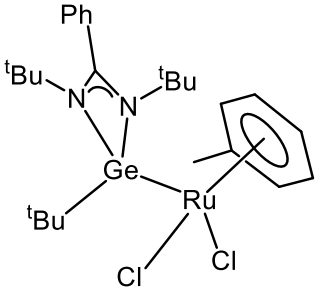

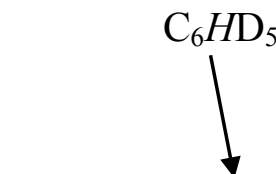

$\mathrm{C}_{6} H \mathrm{D}_{5}$

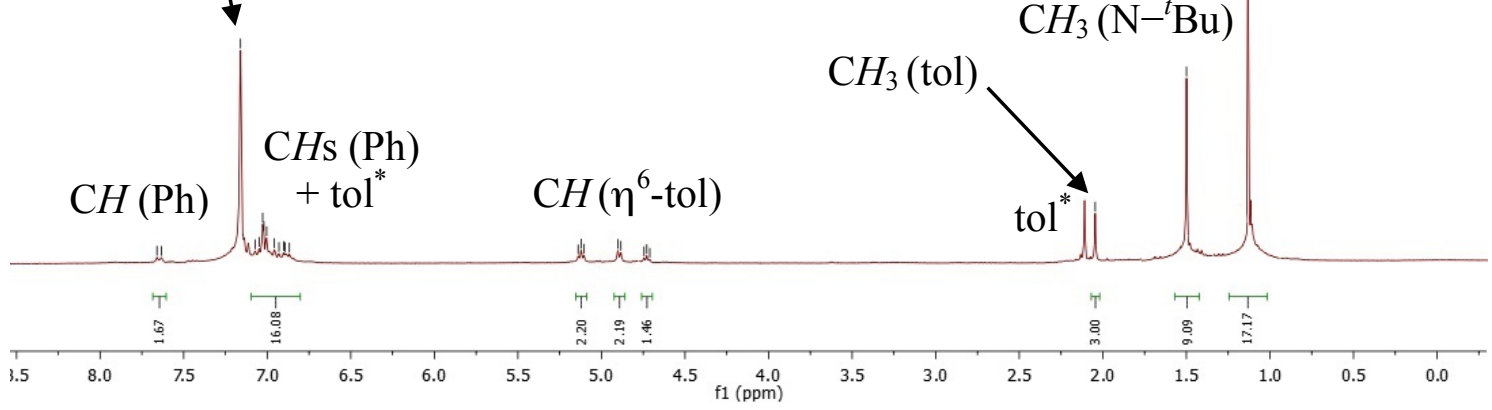

Figure S12. ${ }^{1} \mathrm{H}$ NMR spectra $\left(300.1 \mathrm{MHz}, \mathrm{C}_{6} \mathrm{D}_{6}, 25{ }^{\circ} \mathrm{C}\right)$ of the solid obtained by solvent evaporation after heating complex 5 in toluene at reflux temperature for $24 \mathrm{~h}$. 